\title{
Photographie et cinéma chez Henri Focillon : illustrer, sérier, diffuser et enseigner. Le renouvellement d'une discipline
}

\section{Colin Lemoine}

Volume 31, numéro 1-2, 2006

URI : https://id.erudit.org/iderudit/1069623ar

DOI : https://doi.org/10.7202/1069623ar

Aller au sommaire du numéro

Éditeur(s)

UAAC-AAUC (University Art Association of Canada | Association d'art des universités du Canada)

ISSN

0315-9906 (imprimé)

1918-4778 (numérique)

Découvrir la revue

Citer cet article

Lemoine, C. (2006). Photographie et cinéma chez Henri Focillon : illustrer, sérier, diffuser et enseigner. Le renouvellement d'une discipline. RACAR : Revue d'art canadienne / Canadian Art Review, 31(1-2), 55-63.

https://doi.org/10.7202/1069623ar

\section{Résumé de l'article}

Henri Focillon is representative of a period in art history when the reproducibility of the image was established. The exponential conditions of access to images invite us to rethink art history as a theoretical discipline animated by increasingly operative scholarly analysis. Concreteness, which brings image culture into teaching and the dissemination of knowledge, has determining foundations in photography (reproduction and circulation), in "casting" - lax vehicle and figure of the double - and cinema. Thus, after founding the medieval department of the Musée des moulages in Lyon, Focillon conceived a Bureau International de Reproduction Photographique, a veritable "database" before its time. He also researched "educational" cinema, an enterprise that was symptomatic of a "life of forms," which found in the reproducibility of images a decisive and innovative medium.
Tous droits réservés @ C UAAC-AAUC (University Art Association of Canada | Association d'art des universités du Canada), 2006
Ce document est protégé par la loi sur le droit d'auteur. L'utilisation des services d'Érudit (y compris la reproduction) est assujettie à sa politique d'utilisation que vous pouvez consulter en ligne.

https://apropos.erudit.org/fr/usagers/politique-dutilisation/ 


\title{
Photographie et cinéma chez Henri Focillon : illustrer, sérier, diffuser et enseigner. Le renouvellement d'une discipline.
}

\author{
Colin Lemoine, Mustef. Bourdelle, Paris
}

\begin{abstract}
Henri Focillon is representative of a period in art history when the reproducibility of the image was established. The exponential conditions of access to images invite us to rethink art history as a theoretical discipline animated by increasingly operative scholarly analysis. Concreteness, which brings image culture into teaching and the dissemination of knowledge, has determining foundations in photography (reproduction and circulation), in "casting"- lax vehicle
\end{abstract}

and figure of the double - and cinema. Thus, after founding the medieval department of the Musée des moulages in Lyon. Focillon conceived a Bureau International de Reproduction Photographique, a veritable "database" before its time. He also researched "educational" cinema, an enterprise that was symptomatic of a "life of forms," which found in the reproducibility of images a decisive and innovative medium.
B ologne, 1982. André Chastel y exprima un regret, lors du XXIVe Congrès international d'histoire de l'art. Partant du constat que "les étudiants n'arrivent pas vierges à nos études; ils y arrivent dans un état d'intoxication dû à l'existence et à la diffusion du musée imaginaire, de telle sorte qu'on devrait pendant un an les soumettre à une cure, pour qu'ils oublient ces représentations et ces explications ${ }^{1}$ ", l'historien d'art s'interrogeait de la sorte : "Comment est-il possible que dans nos réunions cet aspect technique fondamental de notre discipline soit complètement ignoré ? Je souhaiterais qu'une section soit obligatoirement consacrée à chaque réunion aux problèmes de la photographie. Il serait souhaitable aussi, me semble-t-il, que dans chaque pays on veuille bien s'interroger sur les premiers cas où la photographie d'une part, les diapositives d'autre part, ont été utilisées pour l'enseignement de l'histoire de l'art ${ }^{2}$." Cette invite fut réitérée par Roland Recht, dans son éditorial d'une récente livraison de la Revue de l'art : "Il faudra un jour tenter d'écrire l'histoire de l'histoire de l'art à la lumière de la photographie $^{3}$. "Ces regrets, dont le premier fut formulé par un des élèves les plus éminents de Focillon, ne pouvait-il être un encouragement à interroger la position de ce dernier lors d'une de ces "réunions ", vingt-deux ans après, à Montréal ?

S'il était une encyclopédie exhaustive de notre discipline, "l'histoire de l'art et la reproductibilité de l'image" ferait à l'évidence l'objet de plusieurs tomes. La pensée d'Henri Focillon - sa généalogie, sa perspicacité mais aussi ses lacunes aurait, vraisemblablement à plus d'un titre, droit de cité en tant que chapitre historiographique majeur. Nous nous efforcerons de rendre compte de la spécificité de ce chapitre en en feuilletant quelques pages essentielles afin, nous l'espérons, de désigner la place et le rôle de Focillon dans une histoire complexe qui vit se renouveler notre champ d'études, à l'époque - et comment ne pas introduire d'ores et déjà Walter Benjamin, ainsi que l'intitulé de cette présente session du Congrès International d'Histoire de l'Art nous y invite ? - de la reproductibilité technique de l'œuvre d'art. Une étonnante locution pourrait ainsi nous introduire dans le vif de notre propos. En effet, le 2 novembre 1928, Focillon, dans une lettre adressée à son homologue et ami roumain Georges Opresco, n'emploie-t-il pas une formule, en apparence sibylline mais riche de sens, lorsque, rassurant son correspondant quant à son projet de musée, il l'invite, résolument optimiste, à enrichir de moulages et d'estampes sa future institution qu'il se plaît à baptiser - et André Malraux semble ne pas être loin - "Musée d'histoire de l'art ${ }^{4}$ " ?

"Musée d'histoire de l'art ". L'expression est révélatrice car elle renseigne sur l'ambivalence, voire le paradoxe, qui réside dans la notion d'" art " depuis les dernières décennies du XIX ${ }^{e}$ siècle. La nouvelle partition muséographique, l'aptitude des chercheurs à fournir des examens synoptiques congruents, la circulation accélérée des informations à l'ère industrielle sont autant de critc̀res pour réévaluer l'art, le diffuser, le transmettre et l'étudier's. L'histoire de l'art est non seulement apte à s'imposer en tant que discipline, puisqu'elle est rendue - désormais et plus que jamais - possible mais surtout, puisque prometteuse de schèmes classificatoires à l'heure de la démocratisation de son objet, nécessaire. Nécessaire, elle l'est en droit. Il sera malaisé qu'elle le soit en fait. La discipline, institutionnalisée précocement - entendons moins tardivement - en Allemagne, connaît en France une gestation chaotique qui - comme l'étude documentée de Lyne Therrien ${ }^{6}$ l'a pertinemment montré - verra la Sorbonne héberger enfin une chaire d'Archéologie en 1876 et d'Histoire de l'art en 1899, à la suite de nombreux atermoiements et résolutions, notamment la création de l'École du Louvre ou de l'École Pratique des Hautes Études?

Ce paragraphe, dense et chaotique, de l'enseignement de l'histoire de l'art en France a néanmoins un intérêt des plus remarquables. Pour parapher la discipline et statucr sur l'autonomie de son existence, il s'agissait d'en définir les critères et d'évaluer ses moyens, quitte à la penser par discrimination. Or, c'est précisément autour de ses moyens que s'articula ce débat substantiel. La gravure, objet de diffusion désormais moins confidentiel, le moulage, interrogeant la sérialité et la fidélité à son original, la photographie récente, promesse d'une vérité de l'image : autant de possibilités accrues de reproduire l'œuvre 
d'art - ou plus exactement l'image de l'œuvre d'art ${ }^{8}$ - et de mesurer la césure ténue entre l'art et les instruments de son étude, étant donné la singularité de ces derniers. La gravure, mécanisée et destinée à l'enseignement, est-elle comparable à la gravure d'un Marc-Antoine? La photographie peut-elle prétendre à être un double du réel? Peut-elle s'ériger en tant que forme artistique à part entière ou n'est-elle qu’un simple véhicule dont il s'agirait de mesurer aussi bien l'efficience que les limites ? Enseigner l'histoire de l'art de 1913 à 1923 à la Faculté des lettres de Lyon, de 1923 à 1938 à la Sorbonne puis au Collège de France et à Yale devait amener Focillon à répondre à ces questions et à leurs enjeux.

Un exemple significatif suffirait à illustrer l'ambivalence de cetre locution de "Musée d'histoire de l'art ". Si nous ne pouvons que souscrire à un postulat affirmant que " beaucoup plus que leur possession, c'est la présence même de ces objets, c'est-àdire le contact direct avec les chefs-d'œuvre qui provoquc [la] recherche [en histoire de l'art] ", nous ne pouvons, par conséquent, que sourire et nous étonner devant l'image, gravée celleci et publiée en 1843, de Désiré Raoul-Rochette, dans la Bibliothèque royale pourvue dc pléthores d'objets et de vitrines saturées d'œuvres, en train de dispenser son enseignement, à un auditoire attentif, en ne désignant du doigt... que les planches d'un manuel illustré 10 ?

En 1913, Focillon est nommé conjointement chargé du cours complémentaire d'histoire de l'art moderne à la Faculté des lettres de l'Université de Lyon et conservateur des musées de la ville. Ses premières fonctions d'enseignant d'histoire de l'art sont donc étroitement liées à celles qu'il remplit à la direction des institutions muséales - Musée des Beaux-Arts et Musée Guimet notamment - et de l'École des Beaux-Arts. Lors de ce "potentat " lyonnais, Focillon nous invite à y revenir : "Musée " et " Histoire de l'art "... Son premier fait d'armes sera la création, suite à celle de la collection d'antiques que l'on doit à Henri Lechat, du Musée des moulages du Moyen Âge, de la Renaissance et des Temps modernes de l'Université de Lyon. En effet, si le Musée des moulages de la Faculté des lettres de Lyon est inauguré en 1899, à la suite et sur l'exemple de ceux de Bordeaux ou Iille, Focillon complétera, en 1913 - date qu'il nous est permis de confirmer définitivement " et qui, étant celle de son arrivée, abonde dans le sens d'une décision au caractère impérieux -, la collection de 1100 moulages réunie par son prédécesseur de pièces prestigieuses à vocation encyclopédique. Complétant ainsi ce qui sera l'une des plus riches collections du genre en France, Focillon devait s'enorgueillir à jamais de cette réalisation, qu'il se plut à rappeler comme étant sienne $e^{12}-$ qui répondait aux périodes qu'il enseigna durant son affectation lyonnaise ${ }^{13}$. Procédant en partie de "la logique métonymique de Viollet-le-Duc " - l'expression est de Dominique de FontRéaul ${ }^{14}$, le Musée des moulages de Lyon, à l'instar de son prestigieux aîné - le Musée de sculpture comparée, créé en 1882 seulement trois ans après qu'il a été formulé -, est un véhicule idéologique mais surtout un outil de diffusion du savoir. Son efficacité permet de plaider en faveur d'un comparatisme, d'une étudc d'après l'œuvre, ou plutôt d'après son double, dans sa tridimensionnalité sérielle et la plus intègre possible, et d'une perspective évolutionniste. Aussi, Louis Courajod et P.-Frantz Marcou, prosélytes œecuméniques, n'hésitaient pas à déclarer sans ambages: "En matière de sculpture, [il n'y a pas] de distinction à faire entre l'œuvre elle-même et sa reproduction ; c'est ainsi que, pour l'histoire de l'art, un moulage, qui ne peut être suspect d'interprétation, a toute la valeur scientifique d'un original et mérite, par suite, d'être examiné avec le même intérêt $^{15}$."

Focillon, moins enfiévré que ces prédécesseurs, ne pouvait néanmoins mésestimer l'importance - non qu'elle fut nouvelle - d'un tel corpus rendu possible en un unique lieu. En effet, avec ses voyages en Italie en vue de la rédaction de sa thèse sur Piranèse, parue en $1918^{16}$, et en tant que regardeur insatiable et directeur des musées de la ville, l'historien d'art s'était formé devant l'œuvre même, autrement dit en sa présence. La consticution d'un tel musée répondait donc parfaitement à un enseignement soucieux de concrétude qui se voyait déployé dans un lieu peuplé de reproductions, laissant entrevoir - il faut ici relire Vie des formes - "les heureuses conséquences qui s'exercent sur la structure, sur la combinaison des masses, sur le rapport des vides et des pleins, sur le traitement de la lumière ${ }^{17} \%$. Le Focillon enseignant était donc un fervent zélateur de ce qu'il nommait " [son] musée des moulages " qui participait d'une foi en un progrès de la reproductibilité des images et d'un besoin impérieux de transmission concrète des connaissances.

Certes, le recul, avec son privilège critique, nous a permis de mesurer les limites idiosyncrasiques et esthétiques d'une telle entreprise ne présentant que ce que nous pourrions caractériser comme des leurres d'œuvre. Roland Recht, partant des propos de Camille Enlart ${ }^{18}$, a parfaitement souligné la latence inhérente à de telles reproductions, identiques en ce point à la photographie : hors contexte, "déshistoricisées" puisque privées de la patine du temps et de l'accident, invalides esthétiquement car réduites à des contrastes lumineux exacerbés, elles ne peuvent par essence - être que des tentatives infructueuses et avortées pour rendre l'exactitude et l'irréductibilité non seulement originelle mais aussi originale de l'œuvre. En ce sens, pour Focillon, les moulages ne sont qu'un étai fécond pour l'enseignement, en aucun cas un substitut de l'original. Ils sont "à l'image de ", inaptes à rendre l'unicité de l'œuvre puisque relevant d'une dématérialité fondamentale, tandis que l'artiste, selon ses mots, est l'homme du geste génésiaque, le pourvoyeur de la présence concrète, commerçant sans cesse avec la pesanteur de l'œuvre : " il touche, il palpe, il suppute le poids, il mesure l'espace, il 
modèle la fluidité de l'air pour y préfigurer la forme, il caresse l'écorce de toute chose, et c'est du langage du toucher qu'il compose le langage de la vue - un ton chaud, un ton froid, un ton lourd, un ton creux, une ligne dure, une ligne molle ${ }^{19}$. " Et Willibald Sauerländer de rappeler : "Henri Focillon, qui parlait de "loi du cadre" dominant l'esthétique de la sculpture romane, était fasciné par l'intégration des sculptures romanes dans l'ordre de l'architecture monumentale. Les moulages, reproductions de fragments isolés, dégagés de leur cadre architectural, permettaient mal de telles observation ${ }^{20}$. "

Les musées spécialisés sont, pour Focillon, une caution scientifique, car s'ils "permettaient mal [des] observations" ainsi que l'affirme l'historien d'art allemand, ils en permettaient néanmoins ou plutôt en dépit de... Ils étaient donc une caution scientifique, certes, mais aucunement suffisante à elle seule, et l'empressement de Focillon à enrichir la collection lyonnaise dès sa nomination à l'université l'atteste - une des possibilités, voire l'une des conditions, de l'émergence de la discipline, subordonnée à la reproductibilité de l'œuvre d'art.

Le moulage, bien que sériel et relativement compétent pour rendre les volumes et les masses, n'en demeurait pas moins un outil encombrant, difficilement accessible et peu enclin à rentrer dans une salle de cours ou un amphithéâtre. Focillon devait nécessairement composer avec l'instrument photographique. Jacob Burckhardt en Suisse et Hermann Grimm, outreRhin, avaient répandu l'usage de la photographie lors de leurs cours d'histoire de l'art. Le second alla jusqu'à formuler dès 1865 auprès du gouvernement prussien un vœu jamais exaucé, celui de la création d'une photothèque : " $\mathrm{Si}$ nous arrivons à composer des bibliothèques de reproductions ptérobranches dans lesquelles ne manque rien de ce qu'il est possible d'avoir, on pourra en effet dans l'avenir parler de l'histoire de l'art moderne comme d'une science solidement fondée ${ }^{21}$. "Toutefois la rareté des sources et des documents, comme le souligne Anthony Hamber ${ }^{22}$, demande à ce que nous spéculions quant aux modalités précises de l'utilisation de la photographie, notamment en France. Mais Focilion ne pouvait ni ne devait se satisfaire des moulages, à une période où l'usage de la photographie était désormais chose admise, ayant perdu en contempteurs et gagné en accessibilité. Wilhlm Vöge, vingt ans avant que Focillon ne soit titularisé à l'Université de Lyon, déclarait de la sorte : " La création du musée des moulages, la constitution d'un trésor de photographies et la reproduction est [...] très certainement un progrès plus grand $[. .$.$] que le meilleur travail critique { }^{23}$."

Aussi, qu'en est-il de la photographie chez Henri Focillon ${ }^{24}$ ? Ce dernier est fils de graveur. Et cette assertion lapidaire suffirait presque, à elle seule, à expliquer non seulement l'origine de son intérêt jamais démenti pour l'estampe et ses possibilités inhérentes de reproduction, mais aussi sa réticence initiale et presque imperméable aux nouveaux procédés techniques, et en premier lieu à la photographie. Rien ne serait plus préjudiciable à l'analyse de la réflexion de l'historien de l'art que de l'abstraire de ce "biotope " familial déterminant. Victor Focillon est un éminent graveur à l'eau-forte, ami de Rodin, Carrière et Geffroy et c'est incidemment que le jeune Henri, en raison d'une forte myopie, renonce à imiter son père pour se consacrer à l'histoire de l'art. Que sa déception ait pu servir d'impulsion à une étude d'autant plus minutieuse des gravures - lui qui ne cessa d'en avoir sous les yeux et sous la main -, il est permis de le croire. Que son père - auquel il consacra des lignes pénétrantes - l'ait influencé, cristallisant son admiration pour la technicité du savoir-faire, il est capital de le souligner.

La gêne de Focillon devant la photographie, qui concurrence la gravure, tient à son effacement du geste artistique. Déjà, Ruskin, animé des mêmes réticences, avait cédé au pouvoir du photographique, ainsi que l'a analysé Wolfgang $M$. Freitag ${ }^{25}$. Pour Focillon comme pour le Britannique, la photographie, dans un premier temps, ne peut être digne d'un Éloge de la main - pour reprendre le titre éponyme de son pamphlet de $1939^{26}$ - puisque, et les mots sont ici empruntés à Benjamin, " avec elle pour la première fois, dans le processus de la reproduction des images, la main se trouve déchargée des tâches artistiques importantes, lesquelles désormais furent réservées à l'œil rivé sur l'objectif ${ }^{2}$ ". L'acharnement anti-photographique de Focillon, à ses débuts, relève plus exactement d'une dénégation au profit de la gravure. Dénégation symptomatique si l'on considère le débat, qui commence uniquement à être étudié en France bien que la tradition anglophone et germanique l'aient soulevé depuis longtemps, et que Trevor Fawcett désigne par l'opposition dichotomique et quasiment binaire du Graphic versus Photographic: "More significant were the growing doubts about the camera's possible effect on aesthetic dicrimination, about its tendency to overemphasize the physical nature of art objects, and about the dangers of accepting the all-too-plausible photograph as if it were the real thing and not a highly reductionist $\operatorname{copy}^{28}$."

Deux textes capitaux permettent d'entendre les arguments de Focillon. En 1911, avec "Les graveurs de la Joconde ${ }^{29}$ ", Focillon spécifie l'originalité de la gravure comme une pratique artistique autonome et examine avec une acuité remarquable les notions d'" authentique " et de "ressemblance " pour convoquer, en négatif, la photographie, "procédé d'une inexactitude si fréquente et si fâcheuse, due en partie à l'ingéniosité des opérateurs ${ }^{30}$ ". La traduction d'un médium à l'autre implique à l'évidence une perte. Quand Henri Delaborde reprochait aux gravures qui tentaient de concurrencer la photographie d'être "inexactement exactes ${ }^{31}$ ", chez Focillon, la perte de la gravure est compensée par un gain - le terme, nous le savons, lui est cher - de "sensibilité", opposant dans un raccourci trouble les talents "personnels" aux talents - et la terminologie est voisine 
d'un Benjamin ${ }^{32}$ - "mécaniques". Pour aller plus loin, l'estampe est un art, la photographie non. Mais certainement Focillon devine-t-il que la question est ailleurs, qu'il transpose en termes esthétiques, en outre bien discutables, ce qu'il conviendrait d'interroger en termes méthodologiques et épistémologiques. D'ailleurs, son article est une forme de compromission, de lapsus visuel, entendons un illogisme révélateur : la planche qui accompagne son propos n'est rien d'autre qu'une photographie reproduisant les gravures invoquées... On le pressent, ce que Focillon blâme est aussi et pourtant une nécessité technique, dont l'uexponentialité" du médium était déjà largement consommée ${ }^{33}$. Aussi Focillon reconnaît-il déjà dans les dernières pages de ce plaidoyer pour l'estampe la spécificité non négligeable de la photographie devenue "certes [...] souhaitable ${ }^{34}$ " mais à laquelle il ne concède pas une primauté sur la gravure, tel Valéry constatant, en 1939, que "le bromure l'emporte sur l'encre ${ }^{35}$."

Ses pages consacrées à "L'eau-forte de reproduction ${ }^{36}$ ", parues un an plus tôt, sont animées de la même idée. Son analyse technique lumineuse de la gravure est suivie de considérations congruentes quant à ses limites et notamment son induction nécessaire, voire dangereuse, d'une interprétation. Les mots vaudraient aussi bien pour la photographie, mais Focillon persévère : "Cette éloquente réduction de valeurs diversement colorées, tâche en face de laquelle la photographie a depuis longtemps fait banqueroute, exige un sens esthétique, un art de choisir et d'interpréter, une science des sacrifices nécessaires qui s'élèvent à la hauteur d'une création proprement dite ${ }^{37}$. „C'est à la lumière de ses allégations que nous pouvons réévaluer l'apparente fantaisie de l'intitulé de son article de 1927 qui, comme pour mieux insister - à la manière d'un négatif - sur la différence substantielle entre photographie et gravure, porte pour titre... " Manet en blanc et noir ${ }^{38}$ ". L'extrême inverse du noir et blanc?

Cependant, il semble que Focillon procède plus à un plaidoyer en faveur de la gravure - résurgence d'une tutelle familiale - qu'à une mise en accusation de la photographie, dont certains commençaient à faire valoir le caractère "respecta$b^{3{ }^{39}}$ ". En dénigrant la photographie, son apparente apesanteur et son caractère éthéré, il tend à asseoir la primauté du geste génésiaque et de la main "ouvrière ". C'est ainsi qu'il ironise dans une lettre à Opresco : "Ce siècle m'épouvante par ses mélanges et par ses interférences [...]. Heureux les types à fond et à forme, les acolytes de Wölfflin et autres purs esthètes! J'ai un étudiant anglais qui m'a proposé des sujets de travaux d'esthétique : j'ai commencé par le couvrir d'injures. Maintenant, nous sommes copains. "À l'instar de cette anecdote, la réconciliation de Focillon avec la photographie - et il nous est permis de douter qu'il y ait eu réellement une brouille - eut bien lieu. En 1922, il précise, à ce même correspondant, que ces conféren- ces à venir, en terre roumaine, seront accompagnées de projections ${ }^{40}$. Et nombreuses sont les références à des photographies dans les lettres de Focillon, qui fut sans cesse prêt à nourrir son répertoire visuel et à s'éduquer iconographiquement. La photographie est médiate et, à ce titre, est un outil de première main puisque, sans être un art, elle a toutefois, pour l'historien de l'art, une valeur éducatrice majeure.

Au premier Congrès international d'histoire de l'art, qui se tint en 1873 à Vienne, fut évoquée la nécessité d'un rapport sur la question d'un matériel d'enseignement, formulation identique au vœu exprimé par André Chastel lors de la XXIVcédition de cette manifestation, en Italie. Inaugural, ce congrès le fut à bien des égards. En effet, Bruno Meyer, futur professeur à l'école polytechnique de Karlsruhe, baptisait solennellement bien que péniblement devant un auditoire aussi sceptique que stupéfait ce qui devait révolutionner l'enseignement de l'histoire de l'art à venir : la plaque de verre, ancêtre de la diapositive. Perplexe, les participants devaient tous recourir à ce procédé quelque vingt ans plus tard, exploitant, dès lors, la reproductibilité de l'image à des fins "grandement " didacriques. D'autant que pour la première fois depuis l'ère de sa reproductibilité, l'image pouvait, à l'envi, être projetée selon différentes dimensions et, précisément, celle de l'objet représenté, optimisant le fantasme de réalité, tout du moins de vraisemblance. Au début des années 1890, Hermann Grimm utilisait le skioptikon ou "lanterne magique", projetant ainsi des images lumineuses à une assemblée interdite devant cette dramaturgie savamment orchestrée : " Le skioptikon nous permet de faire une différence bien nette entre ce qui, dans l'art, touche l'âme et ce qui nous intéresse uniquement sous le rapport de l'histoire de l'art ${ }^{41}$. "

Les témoignages sont maigres concernant la situation en France, mais l'exercice des projections ainsi que l'emploi de supports photographiques destinés à circuler dans les mains des étudiants sont des faits avérés. Salomon Reinach à l'École du Louvre et Achille Luchaire à la Sorbonne étaient des adeptes de la projection ${ }^{42}$, après que Mâle, en 1894, eut insisté sur l'importance de la photographie, arguant de son utilité scientifique. Nous savons aujourd'hui combien l'histoire de notre discipline est tributaire de son matériel, et l'on doit à Malraux, en 1949, d'avoir immortalisé ce passif par sa célèbre formule : "L'histoire de l'art depuis cent ans [...] est l'histoire de ce qui est photographiable ${ }^{43}$." Si la généalogie est délicate, dans cette mythologie complexe de l'histoire de la photographie et de son appropriation, il n'en demeure pas moins que le rythme des innovations alla s'accélérant, écrivant une mythologie de la paternité, de "la première fois": Wölfflin aurait, le premier, projeté ensemble deux images pour mieux donner à voir son discours, tandis que Warburg, en 1912, lors du dixième Congrès international d'histoire de l'art de Rome, aurait le premier joint à sa communication des projections en couleur. 
De son côté - ses lettres et archives l'attestent -, Focillon utilisa la projection pour vraisemblablement chacun de ses cours, à Lyon, notamment pour ceux consacrés à "La Renaissance en France " ou à "L'École espagnole au XVIIe siècle ", aussi bien qu’à Paris. À ce titre, le cours qu'il professa à la Sorbonne sur Piero della Francesca est exemplaire. La vigilance avec laquelle il dispensa ce dernier est telle qu'il se procura un corpus exceptionnel de cent cinquante plaques de verre tant et si bien que ses étudiants purent, à partir des notes rassemblées et de ce " musée imaginaire ", publier un livre posthume sur le peintre d'Arezzo en $1952^{44}$. L'inventaire des plaques de verre que Focillon possédait, de par son ampleur et sa qualité, suffit à signifier son désir d'exploiter les possibilités offertes par la reproductibilité de l'image. Certes, Focillon n'était pas Berenson, dont Nigel Llewellyn rappelle que "son époque est celle de la photographie ${ }^{45}$ ". Et il est d'ailleurs malaisé de distinguer ce que Focillon réservait à son usage strictement personnel. Chose sûre, il n'était pas non plus Ralph Lieberman ou Archur Kingsley Porter qui conjuguèrent pratique de l'histoire de l'art et pratique de la photographie. Kingsley Porter devait d'ailleurs initier, selon Meryle Secrest, le chantre de l'attributionnisme à l'art médiéval lors d'un voyage en Bourgogne ${ }^{46}$. Mais Focillon ne mésestimait pas les études de- Berenson, bien qu'il ne partageât pas son enthousiasme inconditionnel pour la photographie: "Parfois, après une journée à déchiffrer nos monstrueux cailloux du XI siècle, je reprends Les Peintres italiens de la Renaissance, pour respirer l'atmosphère de l'esprit et pour flairer de l'humanité. Vous m'aidez à découvrir l'homme sous la pierre du Moyen Âge ${ }^{47}$. "Car Berenson, qui demanda à être introduit auprès de Focillon ${ }^{48}$, devait composer sa propre photothèque, s'amusant à déclarer : "L'histoire de l'art est un grand jeu de hasard, auquel gagne celui qui possède le plus de photographies ${ }^{49}$. „Et Frederick $\mathrm{N}$. Bohrer, dans sa brillante étude qui convoque l'exemple berensonien, a le mérite de s'interroger sur l'intrication des liens consubstantiels de l'histoire de l'art et de la photographie et de conclure sur une question de propédeutique, d'apparence évidente si elle n'était quelque peu nouvelle tant ce champ d'investigation est relativement récent : "Could there be art history without photography 50 ? "Aussi revient-il sur l'effronterie de Berenson estimant, dans une confession trop peu rapportée malgré l'aveu hautement significatif : "I am not ashamed to confess that I have more often gone astray when I have seen the work of art by itself and alone, than I have knowing its reproduction only ${ }^{51}$."

Pour Focillon, la photographie a une valeur documentaire et didactique. Elle ne remplace pas l'œuuvre. Et heureusement ${ }^{52}$. Disposé aux initiatives de grande envergure, il aura l'occasion d'illustrer son appropriation des nouvelles techniques de reproduction pour l'histoire de l'art. En 1925, il est désigné pour représenter la France, avec Paul Valéry, à la Sous-commission des lettres et des arts au sein de la Société des Nations. C'est à cet effet que, faisant écho aux aspirations de Grimm, vieilles de soixante ans et dont la postérité a été doctement analysée par Heinrich Dilly ${ }^{53}$, ou à celles de Sir Martin Conway, formulées en 1901 dans The Domain of Art - "a great collection of reproductions of all the art of all the world, organically arranged ${ }^{54} "$ - il imagina, ainsi que l'attestent dix-sept feuillets manuscrits, un Bureau international des échanges de reproduction des œuvres d'art, véritable base de données avant l'heure, instituée idéalement par un accord entre des chalcographies et affectée aux échanges de moulages et photographies 55 .

$\mathrm{Si}$, suivant les mots de Walter Benjamin, "la photographie contenait virtuellement le cinéma ${ }^{56}$ ", il manquait au triptyque, moulages-gravures-photographie, un médium.

En septembre 1928, Focillon fut élu membre du conseil d'administration de l'Institut International du cinématographe éducatif ${ }^{57}$ et participa, à Rome, aux deux premières sessions annuelles de la jeune institution avant de s'acquitter de cette tâche face au cumul de ses obligations, ainsi que l'atteste sa correspondance avec Luciano De Feo et Georges Opresco ${ }^{58}$. Ainsi put-il s'enorgueillir auprès de ce dernier : "J'ai fait un effort assez surhumain au Congrès National du Cinéma, dont j'ai fait le rapport général. Je puis dire que je connais la question désormais, du moins pour la France ${ }^{59}$. "C'est dans ce contexte fécond, qui vit Focillon côtoyer Louis Lumière, que le premier rédigea, dans le Bulletin périodique de l'Office des Instituts d'archéologie et d'histoire de l'art, sa capitale " Note sur le cinématographe et l'enseignement des arts ${ }^{60} "$. Bien qu'ayant paru deux ans avant le célèbre "On Movies "61 d'Erwin Panofsky, subtilement analysé par Thomas Y. Levin ${ }^{62}$, l'article de Focillon n'était pas pour autant inaugural dans ce domaine : il convient ici de rappeler que l'article " De la cinéplastique ", souvent passé sous silence, et "Vocation du cinéma " d'Élie Faure datent respectivement de 1920 et $1934^{63}$.

Comme il le fit avec la photographie, Focillon tenta, dans son article, de spécifier le cinéma avant d'en analyser le potentiel, et ce en le distinguant des autres procédés de reproduction : il s'agit d'identifier, si elle existe, la singularité de son surcroît technique ${ }^{64}$. Or, il semble précisément que Focillon crédita le cinéma de facultés séduisantes et opérantes. L'inclusion de la temporalité, du mouvement, de la lumière peut arracher l'étude de l'œuvre à " une sorte de fixité séculaire " et rendre "le plein d'une œuvre " ou encore éviter d'appréhender celle-ci comme " une collection de silhouettes ". À côté de cette vocation documentaire, le film pourrait - car il s'agit bien d'un conditionnel être utilisé à des fins pédagogiques ou, pour reprendre l'épithète consacrée au sein de la Société des Nations, "éducatives ", et notamment dans les écoles d'art. Mais sa gageure principale réside dans la place qu'il conviendrait de lui assigner au sein de l'histoire de l'art. Ses procédés uniques - gros plan, "ralenti " 
ou accélération - lui garantissent une valeur théorique au sein de la discipline. Le cinéma donne à voir. Ainsi, la vie des formes trouverait un moyen de traduction privilégié : "La manière dont [les parties] s'accordent entre elles et s'équilibrent: une succession enchaînée - entendons un "travelling " - peut nous le donner. "L'image de l'œuvre ne serait plus " une concrétion cristallisée à jamais sous la vitrine d'un musée ", mais apte à témoigner de "certains artifices de modelé, des heurts, des brusqueries, ou, au contraire, des passages souples ». La maturité du propos de Focillon est révélatrice de sa capacité de renouvellement. L'aventure du cinéma, pourtant embryonnaire, dans le champ de l'histoire de l'art s'écrivit avec Focillon, moins enclin à sa dénégation initiale de la reproductibilité de l'image : "Quand nous n'avons pas l'œuvre elle-même, mais son image, n'est-il pas souhaitable que cette image, par un artifice bien calculé, se meuve à notre place, devant nous qui restons immobiles?" Et de concéder, plus loin : «La photographie a rendu d'immenses services aux spécialistes de nos recherches ${ }^{65}$."

Vantant, dans le même article, les qualités iconographiques, presque cinématographiques, des planches de l'opus d'un de ses plus brillants élèves - La Stylistique ornementale de la sculpture romane de Jurgis Baltrusaitis -, Focillon anticipait sur l'un de ses propres ouvrages qui nous semble être un parangon en termes de reproductibilité de l'image. En 1938, parut chez Paul Hartmann, dont les prestations éditoriales, notamment quant à la reproduction, ne devaient jamais se démentir, Peintures romanes des églises de France ${ }^{66}$. De Saint-Germain d'Auxerre à Berzé-laville en passant par Saint-Savin-sur-Gartempe, Focillon se livre à un lumineux exercice d'analyse formelle des productions peintes des églises au fil d'une cinquantaine de pages. Fait remarquable, le texte de Focillon est accompagné de cent trente photographies de Pierre Devinoy, qui devait par la suite collaborer à maintes reprises avec Hartmann, notamment pour des publications insignes d'Émile Mâle ou de Sumner McKnight Crosby ${ }^{67}$, élève de Focillon et professeur à Yale qui - et est-ce un hasard ? - sera un temps responsable du Centre audiovisuel de l'université américaine.

Ces photographies, à la différence des vignettes qui jalonnent le texte, ont une valeur non seulement descriptive - elles relèvent bien d'une ekphrasis - mais aussi artistique. En pleine page et d'une qualité graphique extraordinaire, elles sont pourvoyeuses de sens puisque leur splendide noir et blanc ne peut empêcher de niveler certains accidents au profit de la notion, cardinale chez Focillon, de style. Le livre illustré prend ici tout son sens. Mais la collaboration Focillon-Devinoy va plus loin : l'enchaînement savant des photographies permet un déroulement narratif remarquable. Ainsi, la Vierge de Montmorillon, nécessairement coupée par un cadrage rendant justice au $\mathrm{Ma}$ riage mystique de Sainte-Catherine, est intégralement reproduite, à la même échelle, sur la page de vis-à-vis. De même, à côté du
Christ en gloire de Berzé-la-ville, photographié assis dans sa mandorle, la planche voisine ne figure, cette fois, que son visage. Enchaînement de séquences, gros plans, respect de l'échelle: comment ne pas penser au cinéma qui "transforme [...] la simple contemplation en récit ", pour reprendre les mots de Marie-Line Cencig ${ }^{68}$, et qui influença tant Malraux et sa Psychologie de l'art ${ }^{(6)}$ comme l'a savamment démontré Henri Zerner ${ }^{70}$ ? La pertinence scientifique des photographies et leur obsession de la réalité sont telles qu'une page entière est consacrée à des considérations techniques qui légitiment, voire justifient, le parti pris des planches: le matériel, l'angle privilégié, les plaques utilisées et même les conditions atmosphériques sont tour à tour convoqués. La scène séduisante ne devait pas, pour autant, faire oublier les coulisses scientifiques et techniques. Paul Hartmann demanda à Pierre Devinoy de présenter dans un immense tableau l'estimation de la hauteur du sujet ainsi que la distance focale de l'objectif, certainement de manière concertée avec l'historien d'art qui devait suivre le travail du photographe à plusieurs reprises ${ }^{71}$. À cette technicité du savoir-faire, fut ajoutée la description des couleurs des sujets photographiés, nuancier d'une précision exceptionnelle qui permettait de palier au noir et blanc des illustrations. Sans être inédites, ces assertions n'en étaient pas moins rares. Une photographie "artistique " au service d'une cause scientifique et qui emprunte au modèle cinématographique: Focillon exploitait, par syncrétisme, la reproductibilité technique de l'image au sein d'un important ouvrage d'histoire de l'art. Chiasme révélateur de cet enjeu complexe, un dessin de la main de Focillon inversera les rôles : des spectateurs attentifs et disciplinés regardent sur un gigantesque écran de cinéma... un livre d'art - Dessins par un maître inconnu ${ }^{72}$.

Bien loin le temps où, pour louer le médium naissant, un critique peu avisé commenta une photographie de la Joconde par Gustave Le Gray en estimant qu'elle seule pouvait rendre l'ineffable d'une œuvre unique qui s'avéra finalement n'être qu'une pâle reproduction de $1848^{73} \ldots$ À l'inverse, Focillon n'examina jamais cette question en homme lige, malgré sa ferveur. S'il ne ferma jamais les yeux sur les nouvelles techniques de reproduction, il ne s'y aveugla pas non plus. "Focillon l'homme des originaux plus que des plâtres. "La phrase est de Charles Picard $^{74}$. Elle nous rappelle combien Focillon, ami des artistes, directeur des musées de Lyon, connaisseur hors pair des collections d'art européennes, s'éduqua avant tout devant l'œuvre. Instigateur de la section médiévale du Musée des moulages de Lyon, adepte de la projection, promoteur du cinéma éducateur, il ne cessa d'interroger les possibles de la reproductibilité de l'image. "Il ne saurait y avoir de copie absolue, même dans une matière donnée " : sa convocation itérative de la gravure participe de cette prescription. Reproduire nécessite de traduire et de transiger avec un "manque " et un " tempérament ". Décrypter 
et employer avec précaution l'image reproduite est autant une marque de diligence qu'une exaltation : celle de l'unicité de l'œuvre d'art, de son idiosyncrasie ou de ce que Walter Benjamin appellera le " hic et nunc" car - et il semble paraphraser Focillon - "à la plus parfaite reproduction, il manquera toujours une chose $\mathrm{e}^{75}$,.

\section{Notes}

1 André Chastcl, "Sur la communication non-verbale ", Problemi di metodo. Condizioni di esistenza di una storia dell'arte [Atri del XXIV Congresso internazionale di storia dell'arte], Bologne, CLUEB, 1982, p. 253.

2 Ibid., p. 252.

3 Roland Recht, "Histoire de l'art et photographie ", Revue de l'art, $\mathrm{n}^{\circ} 141,2003$, p. 7.

4 "Ce n'est pas pour rien, enfin, que nous avons fondé l'Office des Musées et travaillé les Calchographies, les ateliers de Moulage etc. - Quant aux dessins et aux estampes, notre groupe et vous-même, nous pouvons en inonder le Musée. Pour les tableaux et les objets d'art, nous procéderons d'abord par des prêts. [...] Je ne vous développe pas ce projet pour peser sur votre décision, qui reste libre. Mais rappelez-vous bien que la question du choix ne se pose pour vous qu'après vos conférences. Après cela, vous verrez s'il vaut mieux continuer à servir sur les bords du Léman les intérêts de la paix ou reprendre ici la tradition de l'homme de la Renaissance et servir sur les rives du Danube la pensée universaliste des élites méditerranécnnes. Nous mettrons ces derniers mots en lettres d'or au fronton du Musée d'histoire de l'art. Je vous embrasse fraternellement. " (Lettre de Focillon à Opresco du 2 novembre 1928, rédigée depuis Bucarest, "Lcttres de Henri Focillon à Georges Opresco ", Revue roumaine d'histoire de l'art. Série Beaux-Arts, 1992, t. XXIX, p. 56.)

5 Aussi peut-on s'étonner que Nora Aradi, dans son étude consacrée au rôle des images aux XIX ${ }^{e}$ et XXe siècles au sein de la discipline de l'histoire de l'art, ne fasse jamais allusion ou n'ait jamais recours à la pratique photographique ou, plus généralement, à la diffusion de ces images et aux nouveaux moyens mis en œuvre pour les véhiculer (Nora Aradi, "Le rôle de l'iconographie et de l'iconologie dans les recherches d'histoire de l'art ", Problemi di metodo. Condizioni di esistenza di una storia dell'arte [Atti del XXIV Congresso internazionale di storia dell'arte] Bologne, CLUEB, 1982, p. 201$06)$.

6 Lyne Therrien, L'Histoire de l'art en France. Genèse d'une discipline universitaire [préface par Gérard Monnicr], Paris, Éditions du Comité des travaux historiques et scientifiques.

7 Respectivement en 1882, suite au rapport du 24 janvier par le ministre des Arts Antonin Proust au président de la République Jules Grévy, et en 1869 , après les nombreux rapports du ministre de l'Instruction publique Victor Duruy.

8 Nous renvoyons, quant à cet aspect fondamental, aux pages de Colin Thompson : "Of course reproductions - in practice photographs now - are indispensable, certainly to art history as we know it, and of course they are not completely adequate substitutes for the paintings themselves. " "Why Do You Need to See the Original Painting Anyway?", Visual Resources, t. II, nos 1-2-3, hiver 1981/printemps 1982, p. 22.)

9 Lyne Therrien, L'Histoire de l'art en France. Genèse d'une discipline universitaire, op. cit., p. 41.

10 L'Illustration, 23 décembre 1943, p. 264.

11 Outre les archives qui plaident pour cette date, une étude d'époque sur le milieu incellectuel lyonnais, quc nous avons retrouvée au Musée social de Paris, nous apprend, sans que ne soit nommé Focillon, que « en 1913 naissait un Musée d'art du Moyen Âge, de la Renaissance et des Temps modernes. À ce Musće, s'agrégea en 1917 la bibliorhèque d'Émile Berteaux acquise, pour la Faculté des lettres, par la marquise Arconati Visconti. " (Association française pour l'avancement des sciences, Lyon 1906-1926, Lyon, Société anonyme de l'Imprimerie A. Rey, 1926, p. 43).

12 "Je connais [la belle collection de moulages du Moyen Âge du Louvre] parce qu'elle a été à l'origine de mon Musée de Moulages à la Faculté de Lyon : il y a là-dedans les figures du grand portail de Chartres, le Beau Dieu d'Amiens, etc..." (lettre de Focillon à Opresco du 2 novembre 1928, rédigée depuis Bucarest, "I.ettres de Henri Focillon à Georges Opresco ", op. cit., p. 56).

13 Pour quelques précisions chronologiques, nous renvoyons aux actes Modèles et moulages [Actes de la tablc ronde Lyon, Université Lumière Lyon 2, des 9 et 10 décembrc 1994], Lyon, Éditions de l'Université Lumière Lyon 2, 1994.

14 Dominique de Font-Réaulx, "La redécouverte des collections de moulage ", Le Musée de sculpture comparée. Naissance de l'histoire de l'art moderne [Actes du colloque «Le musée de sculpture comparée : l'invention d'un modèle au XIXe siècle, Paris, Musée des monuments français, 8 et 9 décembre 1999], coll. Idées et débats, Paris, Monum/Éditions du Patrimoine, 2001, p. 28.

15 Louis Courajod et P.-Frantz Marcou, Musée de sculpture comparée. Palais du Trocadéro, Paris, Imprimeric nationale, 1892 [cité par Roland Recht, "Le moulage et la naissance de l'histoire de l'art ", Le Musée de sculpture comparée. Naissance de l'histoire de l'art moderne, op. cit., p. 46].

16 Henri Focillon, Giovanni Battista Piranesi, Paris, H. Laurens, 1918.

17 Id., Vie des formes, Paris, Presses Universitaires de France [réćdition de Vie des formes, Paris, Ernest Leroux, 1934], 2004, p. 14.

18 Roland Recht, "Le Moulage et la naissance de l'histoire de l'art ", Le Musée de sculpture comparée. Naissance de l'histoire de l'art moderne, op. cit., p. 46-53.

19 Henri Focillon, "Éloge de la main ", Vie des formes, op. cit., p. 112

20 Willibald Sauerländer, "Gypsa sunt conservanda : l'obsession de la sculpture comparée ", Le Musée de sculpture comparée. Naissance de l'histoire de l'art moderne, op. cit., p. 76.

21 Hermann Grimm, Über Künstler und Kunstwerke, 1865 [cité par Willibald Sauerländer, "L'Allemagne et la Kunstgeschichte ", Revue de l'art, $\mathrm{n}^{\circ} 45,1979$, p. 6).

22 "The construction of a methodology by those attempting to examine the role played by photography in the study of art during either the last or the present century is an exacting occupation. Art historians rarely mention the photographs they use, and when 
such references do appear, they are seldom deemed worthy of being indexed. " (Anthony Hamber, "The Use of Photography by Nineteenth-Century Art Historians ", Visual Resources, t. VII, fasc. 2-3, 1990, p. 136.)

23 Wilhelm Vöge, Die Anfänge des Monumentalen Stiles im Mittelalter, Strasbourg, Heitz, 1894 [cité par Roland Recht, "Le moulage et la naissance de l'histoire de l'art ", Le Musée de sculpture comparée. Naissance de l'histoire de l'art moderne, op. cit., p. 53].

24 Mentionnons, quant à l'histoire de l'imprégnation de la photographie par l'histoire de l'art, la précieusc analyse de spécial istes dans Art History through the Camera's Lens (Mary Bergstein, dir.), s.l., Gordon and Breach, 1995.

25 Wolfgang M. Freitag, "La servante et la séductrice ", Histoire de l'histoire de l'art, XVIII' et XIX'e siècles [actes des cycles de conférence, Paris, Musée du Louvre, 24 janvier au 7 mars 1994 et 23 janvier au 6 mars 1995I, Paris, Klinsieck et Musée du Louvre, 1997, p. 26568.

26 Henri Focillon, Vie des formes, augmentéc d'un "Éloge de la main ", Bibliothèque de philosophie contemporaine, Paris, Librairie Félix Alcan, 1939.

27 Walter Benjamin, L'CEuvre d'art à l'époque de sa reproductibilité technique (réédition de la $4^{e}$ édition, parue en 1939), Paris, Éditions Allia, 2003, p. 11.

28 Trevor Fawcett, "Graphic versus Photographic in the NineteenchCentury Reproduction ", Art History, Vol. IX, n² 2, juin 1986, p. 207.

29 Henri Focillon, "Les graveurs de la Joconde ", Revue de l'art ancien et moderne, $\mathrm{n}^{\circ} \mathrm{xxx}$, novembre 1911, p. 366-78 [repris sous le titre "La Joconde et ses interprètes", Technique et sentiment. Études sur l'art moderne, Paris, Henri Laurens, 1919].

30 Ibid., 1919, p. 82.

31 Henri Delaborde, Mélanges sur l'art contemporain, Paris, Jules Renouard, 1866, p. 328.

32 Concernant l'influence de l'histoire de l'art sur Walter Benjamin, notamment l'ascendance de Riegl et de Wölfflin sur ce dernier, nous renvoyons à l'étude de Thomas Y. Levin, "Walter Benjamin and the Theory of Art History ", October, t. 47, hiver 1988, p. 77-83.

33 Anthony Hamber a consacré un article capital à cette question : "The Photography of the Visual Arts, 1838-1880", Visual Resources, t. V, 1988-89, p. 289-310 ; t. VI, 1989-90, p. 19-41, p. $165-79$, p. $219-41$.

34 Ibid., 1919, p. 83.

35 Paul Valéry, "Discours", Bulletin de la Société Française de Photographie et de Cinématographie, $81^{\mathrm{e}}$ année, $4^{\mathrm{e}}$ série, $\mathrm{t} . \mathrm{I}, \mathrm{n}^{\circ} 3$, mars 1939.

36 Henri Focillon, "L'eau-forte de reproduction en France ", Revue de l'art ancien et moderne, $\mathrm{n}^{\circ}$ II, 1910, p. 335-50 et p. 437-46 [repris sous le même titre dans Technique et sentiment. Etudes sur l'art moderne, op. cit.].

37 Ibid., 1919, p. 127-28.

38 Henri Focillon, "Manet en blanc et noir ", Gazette des Beaux-Arts, $5^{c}$ année, $\mathrm{n}^{\circ} \mathrm{xVI}$, décembre 1927, p. 332-46 [repris sous le même titre dans Maîtres de l'estampe. Peintres-graveurs, Paris, Librairie Renouard, H. Laurens, 1930].
39 Quant à l'histoire de la légitimation scientifique de la photographie, il convient de renvoyer à l'ouvrage fondamental d'Heinrich Dilly, Kunstgeschischte als Institution : Studien zur Geschichte einer Disziplin, Francfort, Suhrkamp, 1979.

40 " [...] cinq ou six conférences pour chaque série, avec projections, et puis pour les étudiants et les techniciens, quelques entretiens sur nos méthodes ?" (Lettre de Focillon à Opresco du 11 février 1922, "Lettres de Henri Focillon à Georges Opresco ", op. cit., p. 5.)

41 Hermann Grimm, " Die Umgestatlung der Universitätsvorlesungen über neure Kunstgeschichte durch das Skioptikon ", Nationalzeitung, Berlin, 1892, repris dans H. Grimm, Beiträge zur deutschen Kulturgeschichte, Berlin, 1897, p. 307 [cité par Wolfgang M. Freitag, "La servante et la séductrice", Histoire de l'histoire de l'art, XVIII et XIX' siècles, op. cit., p. 274].

42 Sur ce sujet, voir Thomas Ganz, "A history of projection ", History of Photography, v. XIX, $n^{\circ} 3,1995$, p. 273-74.

43 André Malraux, Essais de psychologie de l'art. Le musée imaginaire, Genève, Skira, 1947, p. 32.

44 Henri Focillon, Piero della Francesca, Paris, Armand Colin, 1952.

45 Nigel Llewellyn, "I es connaisseurs", Histoire de l'histoire de l'art, $X V I I I^{e}$ et XIX'c siècles, op. cit., p. 310.

46 Meryle Secrest, Being Bernard Berenson, New York, Holt, Rinehart and Winston, 1979, p. 328-29.

47 Lettre de Focillon à Berenson du 5 janvier 1938, Archives de la villa I Tatti, sans cotc [cité également par Jocelyne Rotily, "Bernard Berenson et les historiens d'art français des années 1920-1940", Mélanges de l'École française de Rome. Moyen Âge, temps modernes, t. 97 (2), 1985, p. 983]. Notons que Jacqueline Rotily, à l'image d'autres études se contentant de mentionner Focillon, qui plus est au regard de la photographie et de ses possibilités accrues d'une prétendue approche formaliste, qualifie de ce dernier ćpithète l'historien d'art français. Or, nous le verrons et l'avons déjà souligné, le problème est complexe. Quoi qu'il en soit, Focillon ne peut être rattaché sans réserve à cette étiquette, pas plus qu'à l'esthétique viennoise et, comme l'auteur l'écrit, aux "principes de Wölfflin " (p. 984).

48 Dans une lettre à Focillon, Louis Gillet lui confie que Berenson, "[...] m'écrit que votre bouquin [L'Art des sculpteurs romans] l'enchante et qu'il veut vous connaître. " (Lettre du 26 avril 1932, Archives Henri Focillon (1881-1943). Paris, Bibliothèque de l'INHA, collection Jacques Doucet, 1998, boîte 25.)

49 Francesco Zeri, Derrière l'image. Conversations sur l'art de lire l'art, Paris, Rivages, 1988, p. 6 [cité par Sylvie Aubenas, Les Usages de la photographie par les historiens de l'art en France, Paris, Presses de l'Université de Paris-Sorbonnc, 2001, p. 63].

50 Frederick N. Bohrer, "Photographic Perspectives: Photography and the Institutional Formation of Art History ", Art History and its Institutions: Foundations of a Discipline, Elisabeth Mansfield, éd., Londres, New York, Routledge, 2002, p. 256.

51 Bernard Berenson, Aesthetics and History in the Visual Arts, New York, Pantheon, 1948, p. 204 [cité par Frederick N. Bohrer, ibid., note 9, p. 258].

52 Il existe une abondante littérature autour de cette question. Nous ne mentionnerons que deux études synthétiques qui ont le mérite 
de faire avec science le tour de la question : celle de Trevor Fawcett ("Art Reproduction and Authenticity", Art Librairies Journal, 1997, vol. XXII, n² 2, p. 20-5) ct cclle, plus datée, de Lorenz Eitner "Art History and the Scnse of Quality", Critiques III, New York School of Art and Architecture, 1974, p. 13-36).

53 Heinrich Dilly, "Das Auge der Kamera und der Kunsthistorische Blick ", Marburger Jahrbuch fur Kunstwissenschaft, t. 20, 1981, p. 81-9.

54 Sir Martin Conway, The Domain of Art, Londres, John Murray, 1901, p. 131.

55 "Note sur un projet de création d'un Burcau intcrnational des échanges de reproductions d'œuuvres d'art ", s.d., 17 feuillets, $A r$ chives Henri Focillon (1881-1943), op. cit., boîte 25.

56 Walter Benjamin, L'Euvre d'art à l'époque de sa reproductibilité technique, op. cit., p. 11.

57 Christel Taillibert, L'Institut International du cinématographe éducatif. Regards sur le rôle du cinéma éducatif dans la politique internationale du fascisme italien [thèse de doctorat: Histoire du cinéma, Paris I, 1998], Paris, L'Harmattan, 1999.

58 Voir respcctivement Archives Henri Focillon (1881-1943), op. cit., boîte 32 et "Lettres de Henri Focillon à Georges Opresco ", op. cit.

59 Lettre du 30 septembre 1931, "Lettres de Henri Focillon à Georges Opresco ", op. cit., p. 74-5.

60 Henri Focillon, "Note sur le cinématographe et l'enseignement des arts ", Bulletin périodique de l'Office des Instituts d'archéologie et d'histoire de l'art, $\mathrm{n}^{\circ} 1$, juillet 1934, p. 42-5.

61 Erwin Panofsky, "On Movies ", Bulletin of the Department of Art and Archaeology of Princeton University, juin 1936, p 5-15 Ipour la première version].

62 Thomas Y. Levin, "Un iconologue au cinéma ", Cahiers du Musée national d'art moderne, t. 59, printemps 1997, p. 34-74.

63 Élie Faure, "De la cinéplastique ", I.a Grande Revue, CIV, n 11 , novembre 1920 , p. 57-72. De son côté, "Vocation du cinéma ", conférence prononcée en 1934, fut publiée dans Le Rôle intellectuel du cinéma (Paris, Institut international de Coopération intellectuelle, $3^{c}$ cahier, 1937) et repris dans le volume posthume Fonction $d u$ cinéma, de la cinéplastique à son destin social (Paris, Plon, 1953).

64 Comme chez Walter Benjamin, la référence focillonienne au théâtre, cn plus de celle à la photographic, est souvent sensible.
65 Henri Focillon, "Note sur le cinématographe et l'cnscignement des arts ", op. cit., p. 42-3.

66 Henri Focillon, Peintures romanes des églises de France, Paris, Paul Hartmann, 1938.

67 Respectivement Notre-Dame de Chartres (Paris, Paul Hartmann, 1948) et L'Abbaye royale de Saint-Denis (Paris, Paul Hartmann, 1953).

68 Marie-Line Cencig, "La description filmique de l'ceuvre d'art ", Le Texte de l'ouvre d'art : la description, Strasbourg, Presses universitaires, Colmar, Musée d'Unterlinden, p. 108.

69 André Malraux, Psychologie de l'art, 3 vol., Genèvc, Skira, 194750.

70 Henri Zerner, "André Malraux ou le pouvoir de la reproduction photographique ", Ecrire l'histoire de l'art, coll. Art et artistes, Paris, Gallimard, 1997, p. 145-67.

71 Lettre de Paul Hartmann à Henri Focillon, à propos de Pierre Devinoy, datéc du 13 octobre 1938, Archives Henri Focillon (18811943), op. cit., boîte 26.

72 Henri Focillon, Dessins pour un maître inconnu, plume, lavis, sépia, n.d., Paris, Fonds Baltrusaitis.

73 L'anecdote est rapportée par Anthony Hamber qui nous rappcllc qu'en 1857 - l'exposition qui vit le dévoilcment du Crystal Palace -, la revue Illustrated London News [ $n^{\circ} 843-44$, vol. XXX, 7 f́́vricr 1857 , p. 121] commentait de la sorte une photographie de Le Gray, représentant le chef-d'œuuve, pour en vanter l'ineffable originalité, alors que cette toile n'était autre qu'une peinture à l'huile d'après Léonard : "setting aside the perfect seizure of the gradations of tint of this picture, we hold it all but impossible for any engraver, however master of his art, to give the sardonic latent smile of this slily beautiful countenance. " (The Use of Photography by Nineteenth-Century Art Historians ", op. cit., p. 143.)

74 Dans sa lettre à Henri Focillon du 7 novembre 1936 (Archives Henri Focillon (1881-1943), op. cit., boîte 26), Charles Picard s'adresse à l'historien d'art par le biais de cette paraphrase éloquente : "Focillon, l'homme des originaux plus que des plâtres, riche en génie, pauvre en galeries."

75 Walter Benjamin, L'CEuvre d'art à l'époque de sa reproductibilité technique, op. cit., p. 13. 